\title{
Bilateral post traumatic thalamic infarct: rare case report
}

\author{
Sumeet Singh, R.S. Mittal, Achal Sharma \\ SMS Medical College, Jaipur, India \\ Dept. of Neurosurgery
}

\begin{abstract}
Bilateral thalamic infarction is rarely seen in cases with artery of Percheron embolization. Artery of Percheron is a rare variant of arterial supply to both thalamus and midbrain. Occlusion of this artery following trauma and causing bilateral thalamic infarction is rare and very few cases are reported in world literature.
\end{abstract}

Key words: Bilateral Thalamic Infarct, Artery of Percheron, MRI.

\section{Introduction}

Occlusion of artery of Percheron is a rare phenomenon and causes bilateral thalamic infarction. It can occur due to embolism to this artery following crush injury of the lower limbs. Occlusion of this artery can be the cause of patient loss of consciousness and must be known to attending neurosurgeon to think of this phenomenon other than infectious, inflammatory or malignant cause.

\section{Case Report}

A 28-year-old man was admitted to Accidental Emergency with history of road side accident. The patient was conscious oriented with GCS 15. CT brain was normal (Figure 1). The initial respiratory and hemodynamic parameters were normal. $\mathrm{He}$ had crush injury of right leg which was highly contaminated. His peripheral pulses of right lower limb were not palpable. Color Doppler shows no flow distal to injured segment. Othopedician performed amputation. After 6hours of the surgery patient becomes unconscious. Within few hours his Glasgow coma score (GCS) decreased to 7 (E2 M4 V1) Patient was shifted to ICU and was ventilated. Medical causes ruled out to know cause of unconsciousness. MRI with DWI done reveals abnormal signal intensity in the paramedian thalami as shown in figures $2,3,4$. DWI confirmed infarction in bilateral thalamic region shown in figure 5. Patient was seen by neurologist who was of the same opinion. After neurology consultation patient was started on intravenous methyl prednisolone 1 $\mathrm{gm} /$ day administered for five days. Ventilation and other supportive treatment were continued. Neurologist advised for thrombolytic agents but could not be started because of amputation done.

After clinical correlation and radiology of the patient a diagnosis of bilateral paramedian thalamic and midbrain infarction due to occlusion of the artery of Percheron by the embolism probably from crushed limb was made. Patient died due to septicemia because of infection of amputated wound. 


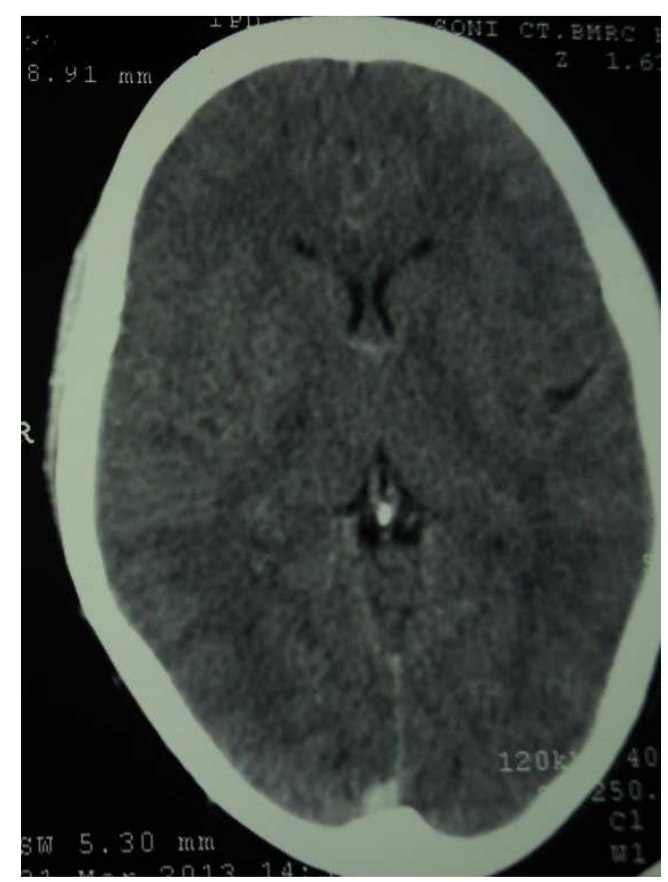

Figure 1 - CT Brain axial image non contrast

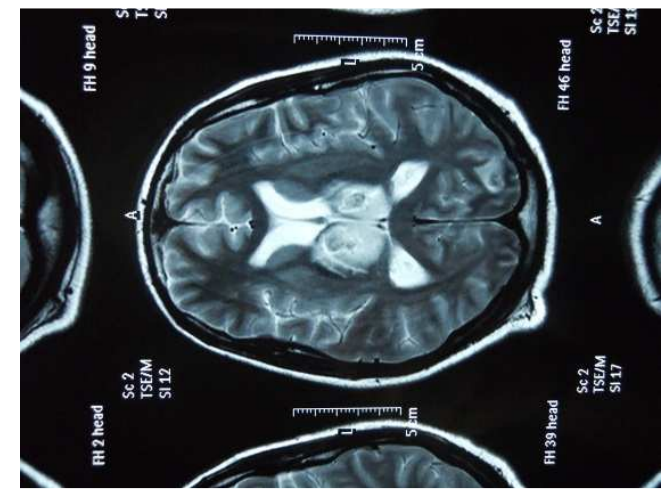

Figure 2 - T2 MRI image showing hyperintensity in bilateral thalamic region

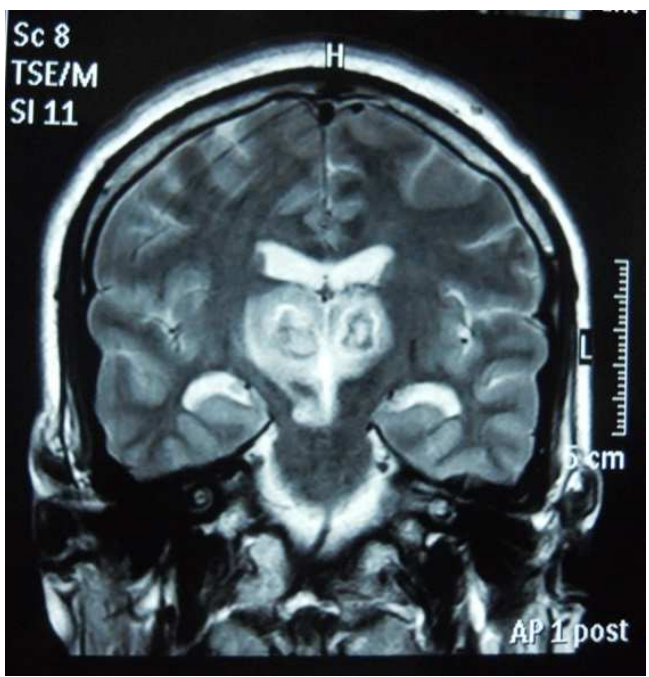

Figure 3 - T2 MRI FLAIR image showing hyperintensity in bilateral thalamic region

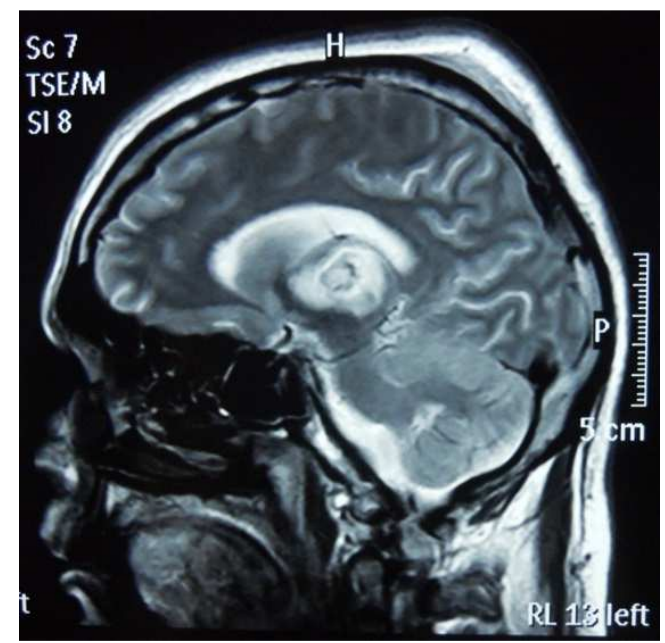

Figure 4 - T1 MRI image showing mixed hypo and hyperintensity in bilateral thalamic region 
DOI: $10.2478 /$ romneu-2014-0065

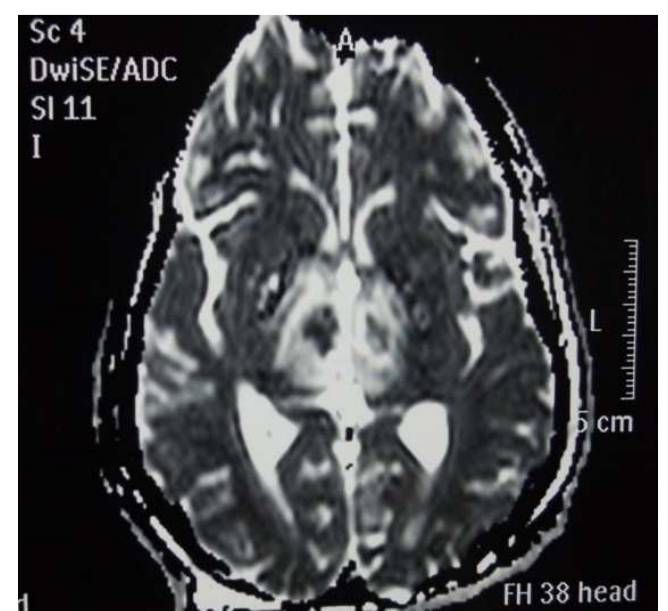

Figure 5 - DWI MRI image showing hyperintensity in bilateral thalamic region

\section{Discussion}

During the 70 s of the past century, G Percheron, the French neurologist (5-7) published three papers describing the vascular anatomy of the posterior circulation, which supplies the human thalamus. According to Percheron, there are four variants of paramedian perforating arteries to the thalami. In most people, these arteries arise from the proximal segments of both posterior cerebral arteries, on each side; this is type I variant. When paramedian penetrating arteries arise directly from the proximal segment of one of the posterior cerebral arteries, type IIa is found. However, in some people, a single arterial trunk stems off the P1 segment of one of the posterior cerebral arteries and this trunk then divides to supply both thalami and the upper midbrain (type IIb); this is the artery of Percheron. Type III is defined by the presence of a single arterial arc that links the proximal segments of both posterior cerebral arteries, and from this arc, the paramedian thalamic perforating arteries arise. Therefore, Percheron artery exemplifies the presence of a small single artery that supplies bilateral vital structures. Bilateral paramedian thalamic infarction with/without midbrain infarction due to involvement of the AOP supplying thalamus is uncommon.

With respect to the prevalence of Percheron artery in the general population, the pertinent literature provides scarce information. Bogousslavsky and co-workers (2) analysed 1000 consecutive patients with first stroke and found that isolated thalamic infarcts as a presenting feature comprised $11 \%$ of all strokes in the posterior circulation while midbrain ischemic infarctions constituted $7 \%$ only. $\mathrm{Uz}$ (8) examined the brains of 15 cadavers; Percheron artery was found in one specimen only. To assess the incidence of bilateral thalamic strokes, Kumral and colleagues (3) studied the registry of 2750 stroke patients; bithalamic infarctions occurred in $0.6 \%$ of their patients only. Lazzaro et al (4) identified four patterns of ischemic infarctions when Percheron artery is occluded. They retrospectively reviewed the clinical presentation and imaging findings of 37 patients with arterial occlusion. Approximately, $43 \%$ of their patients demonstrated damage to both paramedian thalami and midbrain (this was the most common pattern), while $38 \%$ had ischemic damage to paramedian thalami only, without midbrain involvement. Around $14 \%$ of patients, the damage involved the anterior thalamic nuclei in addition to paramedian thalami and upper midbrain. The least common pattern (5\%) was ischemic damage of bilateral paramedian and anterior thalami; the midbrain was spared here.

The sudden loss of consciousness, 
respiratory distress in our patient suggested brainstem stroke. However, the initial brain CT scan of the patient showed normal study. The subsequently done MRI revealed infarction in both paramedian thalami and the left half of the midbrain. The latter observation explains the involvement of artery of Percheron. Prior to his presentation, the patient was conscious; there was no explanation for this young patient's ischemic stroke. To date, through conventional angiography, only four authors were able to visualise this artery (1).

\section{Conclusion}

Artery of Percheron occlusion remains an uncommon event and can be the cause of brain stem stroke and thalamic infarction in patient with crush injury. The initial brain CT scan may be normal and brain MRI imaging shows abnormal signal intensities in the paramedian thalami with/without anterior thalamic or midbrain extension. The treating neurosurgeon should be aware of this condition.

\section{References}

1. A, Wright B, Castillo M, et al. Artery of Percheron infarction: imaging patterns and clinical spectrum. AJNR Am J Neuroradiol 2010; 31:1283-9.

2. Bogousslavsky J, Van Melle G, Regli F. The Lausanne Stroke Registry: analysis of 1,000 consecutive patients with first stroke. Stroke 1988; 19:1083-92.

3. Kumral E, Evyapan D, Balkir K, et al. Bilateral thalamic infarction. Clinical, etiological and MRI correlates. Acta Neurol Scand 2001; 103:35-42.

4. Lazzaro NA, Wright B, Castillo M, et al. Artery of Percheron infarction: imaging patterns and clinical spectrum. AJNR Am J Neuroradiol 2010; 31:1283-9.

5. Percheron G. The anatomy of the arterial supply of the human thalamus and its use for the interpretation of the thalamic vascular pathology. Z Neurol 1973;205:1-13.

6. Percheron G. Arteries of the human thalamus. I. Artery and polar thalamic territory of the posterior communicating artery. Rev Neurol (Paris) 1976;132:297307.

7. Percheron G. Arteries of the human thalamus. II. Arteries and paramedian thalamic territory of the communicating basilar artery. Rev Neurol (Paris) 1976;132:309-24.

8. $\mathrm{Uz}$ A. Variations in the origin of the thalamoperforating arteries. J Clin Neurosci 2007; 14:134-7. 